\title{
La demande d'aide lors des devoirs au secondaire en contextes de classes-portables et traditionnelles
}

\section{HRONIQUE • Recherche étudiante}

\section{Introduction}

Dans cet article, les résultats d'une recherche à la maitrise en didactique à l'Université du Québec à Montréal seront présentés. La recherche porte sur l'impact des technologies sur la stratégie de la demande d'aide et la réalisation des devoirs au secondaire. Ce texte traitera de la problématique, du cadre théorique et de la méthodologie de l'étude. Il exposera finalement les résultats obtenus et leur portée.

\section{Problématique}

Les enseignants de partout dans le monde donnent des devoirs à leurs élèves. Dans une synthèse des écrits, Cooper, Robinson et Patall (2006) affirment clairement que ces tâches à réaliser en dehors des heures de cours permettent la réussite scolaire à condition de les terminer. Selon une vaste enquête de Karsenti (2015), seulement $30 \%$ des 5744 élèves québécois interrogés affirment faire tous leurs devoirs. Plusieurs décident d'abandonner devant les difficultés plutôt que de demander de l'aide.

La recherche d'une assistance, en personne ou en ligne, représente pourtant une stratégie possiblement nécessaire à la réalisation d'un devoir (Puustinen, 2013). Cependant, elle peut susciter des émotions négatives et être perçue comme une menace à l'autonomie ou à l'estime personnelle. L'évitement de la sollicitation d'un soutien s'explique, entre autres, par la peur de paraitre dépendant ou ignorant (Newman,1998). Des recherches 
montrent que l'intégration des technologies en salle de classe diminue ces sentiments négatifs, car elle modifie les relations avec l'enseignant ou avec les pairs, elle permet une plus grande accessibilité aux ressources et elle assure le développement de compétences technologiques. Ces contextes favorisent par conséquent une plus grande qualité et quantité de demandes d'aide (Kitsantas et Chow, 2007).

Les classes-portables, où chaque élève possède son propre ordinateur en cours et à la maison, représentent l'un de ces contextes de classes soutenues par les technologies. À notre connaissance, il n'existe jusqu'à ce jour aucune recherche qui porte sur la demande d'aide dans des classes-portables. La présente étude cherchait donc à répondre à la question suivante : est-ce que des élèves de classes-portables diffèrent d'élèves de classes traditionnelles quant aux menaces perçues associées à la demande d'aide, aux types de demandes d'aide utilisés et à la quantité de devoirs terminés au secondaire?

\section{Cadre théorique}

Depuis la fin des années 80, la recherche d'une assistance pour atteindre un but tel que la réalisation d'un devoir est reconnue comme une stratégie d'autorégulation nécessaire à la réussite scolaire (Zimmerman et Martinez-Pons, 1986). Le modèle sociocognitif de l'autorégulation de Zimmerman (2000) permet de saisir la complexité du concept de la demande d'aide. L'utilisation de cette stratégie est le résultat d'influences personnelles et environnementales. La qualité de la demande d'aide dépend ainsi des menaces perçues à l'égard de cette stratégie et des caractéristiques de l'environnement de la classe (Newman, 1998).

Selon le modèle sociocognitif de l'autorégulation, le contexte de la classe-portable modifierait positivement les perceptions et les habiletés des apprenants. En effet, en plus de faciliter l'accès aux ressources en ligne, les projets portables impliquent un enseignement explicite des compétences technologiques nécessaires à la recherche d'une assistance en ligne de façon adéquate. Ils encouragent également des activités d'apprentissage centrées sur l'élève qui modifient positivement les relations avec les pairs et avec l'enseignant (Zheng, Warschauer, Lin et Chang, 2016).

\section{Méthodologie}

L'échantillon de la recherche se composait de 142 élèves de cinquième secondaire de deux écoles du Québec. Dans la première, les élèves évoluaient dans des classes-portables. Dans la deuxième, les élèves n'utilisaient pas d'outils technologiques en classe. Ce contexte reçoit l'appellation de classe traditionnelle dans la présente étude. Les participants ont dû répondre à un questionnaire construit à l'aide d'items sur les menaces perçues et la demande d'aide issus de recherches récentes (Reeves et Sperling, 2015; White, 2011). Des tests $t$ ont permis de comparer les résultats obtenus dans les deux contextes de classes.

\section{Résultats}

Les deux types de classes diffèrent quant à l'utilisation des outils technologiques pour faire les devoirs. Sans surprise, les élèves des classes-portables $(M=3,5, E T=1,13)$ utilisent davantage leurs outils que ceux des classes traditionnelles $(M=3,03, E T=1,17) ; t(140)=-2,17, p=, 03$. De façon générale, les 
menaces perçues associées à la demande d'aide sont équivalentes pour les deux groupes. Néanmoins, les élèves des classes traditionnelles $(M=2,14, E T=1,14)$ perçoivent plus négativement la sollicitation du soutien de l'enseignant comparativement aux apprenants des classes-portables $(M=1,77, E T=, 92)$; $t(140)=-2,01, p=, 95$.

De plus, les élèves des classes-portables optent davantage pour la recherche d'une assistance en personne et en ligne que les apprenants des environnements traditionnels, que ce soit de façon adéquate ou non. Effectivement, ils tendent davantage à utiliser des stratégies inappropriées, telles que de copier la réponse d'un devoir en ligne $(M=2,29, E T=, 93)$, lorsque comparés aux apprenants des milieux traditionnels $(M=1,84, E T=, 72) ; t(140)=-3,06, p=, 003$. Ces derniers montrent une plus grande propension à éviter de solliciter un soutien $(M=2,92, E T=, 99)$ par rapport aux apprenants des classes-portables $(M=2,48, E T=, 73) ; \mathrm{t}(140)=2,92, p=, 004$.

Finalement, les élèves des classes-portables $(M=3,68, E T=, 97)$ terminent une plus grande quantité de devoirs que ceux issus des classes traditionnelles $(M=2,68, E T=1,18) ; t(140)=-4,76, p<, 001$. Selon la taille d'effet calculée, la différence entre les deux groupes est considérable $\left(\eta^{2}=, 14\right)$.

\section{Discussion}

Les résultats obtenus permettent de croire que l'implantation de classes-portables atténue les menaces perçues associées à la demande d'aide et encourage l'utilisation de cette stratégie lors de la réalisation des devoirs. Par conséquent, les apprenants évoluant dans ces contextes soutenus par les technologies terminent plus souvent les tâches à faire en dehors des heures de cours. Néanmoins, un enseignement explicite des stratégies adéquates de demandes d'aide reste nécessaire afin de prévenir le mésusage des ressources en ligne et d'assurer un effet à long terme sur la réussite scolaire.

\section{Conclusion}

En somme, l'intégration des technologies en classe a un impact bénéfique sur la sollicitation d'un soutien et la réalisation des devoirs. Une future recherche pourrait vérifier si certaines caractéristiques des classes-portables (accès aux ressources, relation avec l'enseignant ou les pairs) expliquent la diminution des menaces et l'utilisation de la demande d'aide.

\section{Références}

Cooper, H., Robinson, J. C. et Patall, E. A. (2006). Does homework improve academic achievement? A synthesis of research, 1987-2003. Review of Educational Research, 76(1), 1-62. http://dx.doi.org/10.3102/00346543076001001

Karsenti, T. (2015). Quel est le rôle d'Allô prof dans la persévérance et la réussite scolaires des élèves? Étude auprès de 6659 acteurs scolaires (élèves, enseignants, directions et parents). Repéré à http://www.karsenti.ca/alloprof/files/Rapport.pdf

Kitsantas, A. et Chow, A. (2007). College students' perceived threat and preference for seeking help in traditional, distributed, and distance learning environments. Computers E' Education, 48(3), 383-395. http://dx.doi.org/10.1016/j.compedu.2005.01.008

Newman, R. S. (1998). Adaptive help seeking: A role of social interaction in self-regulated learning. Dans S. A. Karabenick (dir.), Strategic help seeking: Implications for learning and teaching (p. 13-37). Mahwah, NJ : Lawrence Erlbaum Associates, Inc. 
Puustinen, M. (2013). La demande d'aide chez l'élève : avancées conceptuelles, méthodologiques et nouvelles données. Paris : L'Harmattan.

Reeves, P. M. et Sperling, R. A. (2015). A comparison of technologically mediated and face-to-face help-seeking sources. British Journal of Educational Psychology, 85(4), 570-584. http://dx.doi.org/10.1111/bjep.12088

White, M. C. (2011). Predicting success in teacher certification testing: The role of academic help seeking. The International Journal of Educational and Psychological Assessment, 7(1), 24-44. Repéré à https://files.eric.ed.gov/fulltext/ED536706.pdf

Zheng, B., Warschauer, M., Lin, C.-H. et Chang, C. (2016). Learning in one-to-one laptop environments: A meta-analysis and research synthesis. Review of Educational Research, 86(4), 1052-1084. http://dx.doi.org/10.3102/0034654316628645

Zimmerman, B. J. (2000). Attaining self-regulation: A social cognitive perspective. Dans M. Boekaerts, P. R. Pintrich et M. Zeidner (dir.), Handbook of self-regulation (p. 13-39). San Diego, CA : Academic Press. http://dx.doi.org/10.1016/B978-012109890-2/50031-7

Zimmerman, B. J. et Martinez-Pons, M. (1986). Development of a structured interview for assessing student use of selfregulated learning strategies. American Educational Research Journal, 23(4), 614-628. http://dx.doi.org/10.3102/00028312023004614

\section{Pour citer cet article}

Bisaillon, J. (2019). La demande d'aide lors des devoirs au secondaire en contextes de classes-portables et traditionnelles. Formation et profession. 27(3), 113-116. http://dx.doi.org/10.18162/fp.2019.a189 